\title{
Localizability of unicycle mobiles robots: an algebraic point of view.
}

\author{
Hugues Sert, Wilfrid Perruquetti, Annemarie Kokosy, Xin Jin, Jorge Palos
}

\begin{abstract}
A single landmark based localization algorithm for unicycle mobile robots was provided in [1]. It is based on the algebraic localizability notion and an efficient differentiation algorithm in noisy environment ([2], [3]). Let us stress that this localization algorithm do not need to know the linear and the angular velocities which are reconstructed by this algorithm using the kinematic model. In this paper, a sensibility study leads to a new fusion algorithm in the multi landmark case using as a basis our posture differentiation based estimator. Some simulations and experimental results are presented in order to prove the effectiveness of the proposed method compared to the well known EKF method.
\end{abstract}

\section{INTRODUCTION}

In order to perform a navigation task, the localization algorithm should provide an estimate the robot's posture [4]. It can be either a relative position estimation or an absolute position estimation (see [5]). The relative approach is suffering from the drift problem (odometry or IMU) which can be compensated by fusing data ([6] and [7] fuses IMU data with GPS ones). The absolute approach relies on external measurements with respect to features which are called landmarks (points owith known coordinates: active or natural). Several works deal with active landmarks as in [8] (wifi infrastructure), [9] (bluetooth protocol), and [10] (magnetic patterns). The main drawback of these methods is that they need environment modifications to work. To use natural points, three kinds of sensors are particularly suitable: laser scanners, ultrasound sensors or cameras. The first two ones give distance information, the third one gives angular information. For a monocular camera, some natural points have to be extracted from the video stream: corner points ([11], [12]) can be used and other algorithms are suitable such as SIFT [13] or SURF [14]. This aspect is not dealt in this paper but is considered as an input of the proposed work. The main limitation using the monocular approach is that the distance information is lost during the acquisition process. One solution is to use several points and to estimate the position by triangulation [15]. However, in some situations it can be difficult to find enough landmarks. With only one landmark, a scale factor has to be computed

Hugues Sert, Annemarie Kokosy, Xin Jin and Jorge Palos are with ISEN, 41 bvd Vauban, 59046 Lille Cedex, France (e-mail: hugues.sert@isen.fr, annemarie.kokosy@isen.fr, xin.jin@isen.fr, jorge.palos@isen.fr).

Wilfrid Perruquetti and Hugues Sert are with Non A INRIA - LNE, Parc Scientifique de la Haute Borne 40, avenue Halley Bt.A, Park Plaza 59650 Villeneuve d'Ascq and LAGIS UMR CNRS 8219, Ecole Centrale de Lille, BP 48, Cité Scientifique, 59651 Villeneuve-d'Ascq, France (e-mail: wilfrid.perruquetti@inria.fr).

This work was partially supported by INTERREG IV A 2 Mers Seas Zeen Cross-border Cooperation Program 2007-2013 under SYSIASS 6-20 project - LNE, Mission Insertion Thales, Fondation Norbert Ségard and the Non-A INRIA. in order to get this distance information: the scale factor can be derived from the shape of the landmark [16], [17], [18] or from the robot's motion. For example, [19] and [20] use an odometer to get the distance traveled by the robot between two measures: this method is affected with the drift drawback.

A common framework to study the robot's localizability is related to the observability problem in the sense of the automatic control community (see [21], [22], [23]). Since [24] proves that each non degenerate wheeled mobile robot belongs to one of the five classes of robots and that the possible outputs come from two main classes of sensors (distances and angles), the general problem has a low combinatoric possibility. This is the chosen approach in this paper. In section II, the notations are given. Then, localizability is defined and connected to the observability notion in section III. Section IV provides a sensibility study of the single landmark based localization algorithm from the authors ([1]). This sensibility study leads to a new fusion algorithm in the multi landmark case. Finally, in section V, simulations and experimental results show the effectiveness of the proposed method.

\section{NOTATIONS}

In this paper the following notations are used (see Fig. 1):

- $x^{T}$ denotes the transpose of the vector $x$.

- $P=(x, y, \theta)^{T}$ is the robot's posture (position and orientation).

- $\left(x_{A_{i}}, y_{A_{i}}, z_{A_{i}}\right)^{T}$ are the coordinates of the landmark $A_{i}$. Then using the relative coordinates robotlandmark: $x_{r, i}=x_{A_{i}}-x, y_{r, i}=y_{A_{i}}-y$, the distance between the robot and the landmark are: $d_{3 D}\left(R, A_{i}\right)=\sqrt{x_{r, i}^{2}+y_{r, i}^{2}+z_{A i}^{2}}$ in the space, $d_{2 D}\left(R, A_{i}\right)=\sqrt{x_{r, i}^{2}+y_{r, i}^{2}}$ in the motion plane.

- for a physical variable $z: z_{m}$ denotes its measured quantity (usually $z_{m}=z+\varpi_{z}$ where $\varpi_{z}$ is an additive noise) and $z_{f}$ denotes its filtered value and $\hat{z}$ denotes its estimate.

\section{LOCALIZABILITY}

In order to define and characterize localizability, we first need to specify the robot kinematic model (subsection III-A) and the measurement model (subsection III-B). Then localizability is defined and related to observability (subsection III-C). 


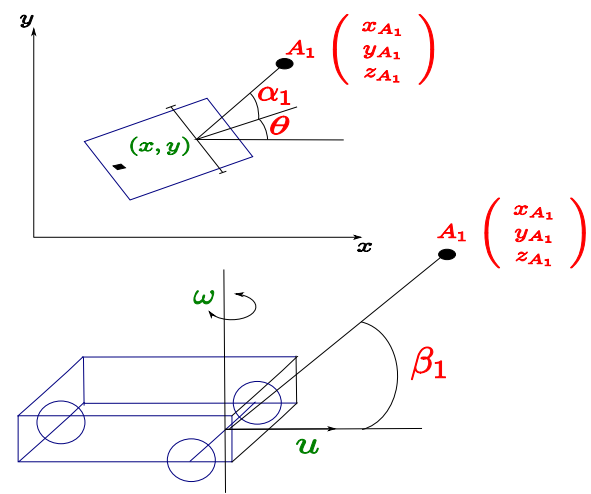

Measured Estimated

Fig. 1. Robot and landmark notation for the localization

\section{A. Robot State Model}

The unicycle mobile robot is fully described its posture $\mathbf{x}=(x, y, \theta)^{T}$ where $(x, y)^{T}$ are the coordinates of the midpoint between the two driving wheels and $\theta$ is the orientation angle with respect to a fixed frame. Its kinematic model is:

$$
\dot{\mathbf{x}}=\mathbf{f}(\mathbf{x}, \mathbf{v})=\left(\begin{array}{c}
u \cos (\theta) \\
u \sin (\theta) \\
\omega
\end{array}\right)
$$

where is the of the robot and $\mathbf{v}=(u, \omega)^{T}$ is the control input (linear velocity, angular velocity).

\section{B. Measurement Model}

This robot observes landmarks in 3D and measures their azimuth and elevation angles:

$$
\begin{aligned}
\mathbf{y}_{i} & =\left(\begin{array}{c}
\alpha_{m, i} \\
\beta_{m, i} \\
\theta_{m}
\end{array}\right)=\left(\begin{array}{c}
\alpha_{i} \\
\beta_{i} \\
\theta
\end{array}\right)+\left(\begin{array}{c}
\varpi_{\alpha_{i}} \\
\varpi_{\beta_{i}} \\
\varpi_{\theta}
\end{array}\right), \\
\left(\begin{array}{c}
\alpha_{i} \\
\beta_{i}
\end{array}\right)= & \left(\begin{array}{c}
\arctan \left(\frac{y_{r, i}}{x_{r, i}}\right)-\theta \\
\left.\arctan \left(\frac{z_{A_{i}}}{\sqrt{\left(x_{r, i}\right)^{2}+\left(y_{r, i}\right)^{2}}}\right)\right),
\end{array}\right.
\end{aligned}
$$

see notations II: additive noises are denoted by $\varpi_{\alpha_{i}}, \varpi_{\beta_{i}}$ and $\varpi_{\theta}$

\section{Localizability}

1) Observability vs. Localizability: Let us formulate our landmark based localization problem as an observability. For this, let us write the state model under the following form (see [24]):

$$
\left\{\begin{array}{l}
\dot{\mathbf{x}}=\mathbf{f}(\mathbf{x}, \mathbf{v}) \\
\mathbf{y}=\mathbf{h}(\mathbf{x})+\varpi
\end{array},\right.
$$

where $\mathbf{x} \in \mathcal{X}$ is the state vector, $\mathbf{u} \in \mathcal{V}$ is the control input vector, $\mathbf{y} \in \mathcal{Y}$ is the measurement vector, $\varpi$ is the noise measurement and $\mathcal{X}, \mathcal{U}, \mathcal{Y}$ are differential manifolds of dimension respectively $n, m$ and $p$ (thus their tangent spaces are diffeomorphic respectively to $\mathbb{R}^{n}, \mathbb{R}^{m}$ and $\mathbb{R}^{p}$ ).
On one hand an observability problem roughly consists in estimating the vector state from the measured data (outputs and inputs). On the other hand for a robot, the localizability problem roughly consists in estimating the posture state from the measured data (outputs and inputs). Usually, the posture vector is a subset of the state vector (see for example the car's kinematics), so the localizability problem is a partial observability problem.

Definition 1: A robot is localizable if and only if its posture vector is observable with respect to a given measurement model.

Note 1: For a unicycle robot, the state vector is the posture vector, so the localizability problem and the observability problem are the same.

2) Review of the observability in the differential geometric framework: In a differential geometry framework, a local weak observability has been introduced in the literature and was characterized by a sufficient condition obtained by Hermann and Krener in [25]: the local observability rank condition (similar, in some sense, to the well known Kalman rank criterion for linear system).

Theorem 1 (Herman-Krener): System (4) is locally weakly observable at a point $\mathbf{x}_{0} \in \mathcal{X}$, if there exist $\mathcal{X}\left(\mathbf{x}_{0}\right)$ an open neighborhood of $\mathbf{x}_{0}$ and positive integers $i_{1}, i_{2}, \ldots, i_{m} \quad\left(i_{j}\right.$ is called the observability indices of $j^{\text {th }}$ output) satisfying $\sum_{j=1}^{m} i_{j}=n$ such that, $\forall \mathbf{x} \in \mathcal{X}\left(\mathbf{x}_{0}\right): \operatorname{dim} d \mathcal{O}_{\mathbf{x}_{0}}(\mathbf{h})=n . d \mathcal{O}_{\mathbf{x}_{0}}(\mathbf{h})$ called the co-distribution of observability is the set of row vectors defined by $\left\{d L_{\mathbf{f}}^{i-1} \mathbf{h}_{j}(\mathbf{x}): j=1, \ldots, m ; i=1, \ldots, i_{j}\right\}$, where $L_{\mathbf{f}}^{i-1} \mathbf{h}_{j}(\mathbf{x})$ are recursively defined by $\left\{L_{\mathbf{f}}^{i} \mathbf{h}_{j}(\mathbf{x})=\right.$ $L_{\mathbf{f}}\left(L_{\mathbf{f}}^{i-1} \mathbf{h}_{j}(\mathbf{x})\right)$ and $L_{\mathbf{f}}^{0} \mathbf{h}_{j}(\mathbf{x})=\mathbf{h}_{j}(\mathbf{x}), L_{\mathbf{f}} \mathbf{h}_{j}(\mathbf{x})=\frac{\partial \mathbf{h}_{j}}{\partial(\mathbf{x})} f$ is the Lie derivative of the scalar function $\mathbf{h}_{j}$ along the vector field $\mathbf{f}$ where $u$ is frozen ( $u$ is considered as a constant)

Remark 1: Let us note that in fact $L_{\mathbf{f}}^{k} \mathbf{h}_{j}(\mathbf{x})$ is the $k^{t h}$ time derivative of the output $h$ along the vector field $\mathbf{f}$.

Theorem 2: A mobile robot with the state dynamics (4) is localizable if, and only if, the dimension of the codistribution of observability, $d \mathcal{O}_{\mathbf{x}}(\mathbf{h})=\mathcal{O}(d x, d y, d \theta)^{T}$ is 3 .

3) Review of observability in the algebraic framework: Since local observability rank condition is concerned with the successive output time derivatives (see proposition 1 and remark 1), it is interesting to look at observability in a differential algebraic framework (see [26], [27]).

Theorem 3 (see [26] and [27]): A system variable $\mathrm{x} \in$ $\mathcal{X}$ is said to be observable if, and only if, it is algebraic over $\mathbf{f}\langle\mathbf{v}, \mathbf{y}\rangle$. An input-output system $\mathcal{X} / \mathbf{f}$ is said to be observable if, and only if, the extension $\mathcal{X} / \mathbf{f}\langle\mathbf{v}, \mathbf{y}\rangle$ is algebraic. $\mathbf{f}$ is the ground field (in practice either $\mathbb{R}$ or $\mathbb{C}$ ).

This differential algebraic criterium can be rephrase in a down to earth formulation as follows: a system variable $\mathbf{x} \in \mathcal{X}$ is observable, if and only if there is an algebraic equation linking $\mathbf{x}$, the outputs, the inputs and a finite number of the time derivatives of the inputs and outputs. Let us use this algebraic criterion for our localization problem.

Theorem 4: A mobile robot with the state dynamics (4) is localizable if, and only if, there is an algebraic equation 
linking the posture to the measured output $\mathbf{y}$, the input $\mathbf{v}$ and a finite number of their time derivatives.

Let us consider the kinematic model of

Example 1: For the mobile robot (1) with measured output $\mathbf{y}=(x, y)^{T}$ we have:

Differential Geometry: Since $d \mathcal{O}_{\mathbf{x}_{0}}(\mathbf{h})=\operatorname{span}\{d x, d y, d \theta\}$ (in fact $\operatorname{span}\{d x, d y, d(u \cos (\theta)), d(u \sin (\theta))\}$, thus $\operatorname{dim} d \mathcal{O}_{\mathbf{x}_{0}}(\mathbf{h})=3$ which means that a unicycle type mobile robot is localizable.

Differential Algebra: Since $x=x, y=y, \tan \theta=\frac{\dot{y}}{\dot{x}}$ we obtain the same conclusion and an algorithm to reconstruct the posture vector (a zero order differentiator on measures to filter $x$ and $y$ and a first order differentiator on measures to estimate $\theta$ ).

So the localizability problem is clearly linked to the differentiation in noisy environment problem. A numerical differentiation algorithm of noisy signals was introduced in [28], analyzed in [2], [3] and recalled here for a measured signal $z_{m}=z+\varpi_{z}\left(\varpi_{z}\right.$ is the noise $)$.

a) Continuous time version of the derivative: The $n^{\text {th }}$ time derivative estimate of the variable $z$ is given by:

$$
z_{f}^{(n)}(T t ; \kappa ; \mu ; N)=\int_{0}^{1} g_{\kappa, \mu, N}(\tau) z_{m}(t-\tau) d \tau,
$$

where $z_{m}$ is the measured quantity of $z$ (see notations) and

$$
g_{\kappa, \mu, N}(\tau)=\sum_{l=0}^{q} \lambda_{l} h_{\kappa+q-l, \mu+l}(\tau)
$$

with $(\kappa ; \mu) \in \mathbb{N}, q=N-n, p=n+\kappa, l=0, \ldots, q$ and

$$
\begin{aligned}
\lambda_{l} & =(-1)^{q-l}\left(\begin{array}{c}
p+q-l \\
p
\end{array}\right)\left(\begin{array}{c}
p+q+1 \\
l
\end{array}\right), \\
h_{\kappa, \mu}(\tau) & =\frac{(-1)^{n} \gamma_{\kappa, \mu, n}}{T^{n}} \operatorname{rect}(\tau) \frac{d^{n}}{d \tau^{n}} \omega_{\kappa, \mu}(\tau) \\
\operatorname{rect}(\tau) & =\left\{\begin{array}{c}
1 \text { if } \tau \in[0 ; 1] \\
0 \text { ortherwise }
\end{array}\right. \\
\omega_{\kappa, \mu}(t) & =t^{\kappa+n}(1-t)^{\mu+n} \\
\gamma_{\kappa, \mu, n} & =\frac{(\mu+\kappa+2 n+1) !}{(\mu+n) !(\kappa+n) !}
\end{aligned}
$$

See [2] for details about the choice of the different parameters involved in this differentiation estimation method.

Remark 2: Let us note that this formula is still valid for $n=0$ and thus gives a filtered estimate of the measured variable. This will be used to obtain the following filtered quantities: $\alpha_{f, i}, \beta_{f, i}, \theta_{f}, \dot{\alpha}_{f, i}, \dot{\beta}_{f, i}$.

b) Discrete version: The $n^{\text {th }}$ order time derivative estimation in the discrete can be obtained by using a numerical integration in Eq. (5). The $n^{t h}$ order time derivative estimation in the discrete case is obtained as the output of the finite impulse response filter (FIR):

$$
\widehat{z}^{(n)}(t-T \xi ; \kappa, \mu, q) \approx \sum_{j=0}^{M-1} W_{j} g_{j} z_{m, l-j},
$$

where $z_{m, l}=z_{m}\left(l T_{s}\right), T_{s}$ is the sampling period, $M$ is the number of coefficients of the filter and it is related to the length of the integration time interval by $T=M T_{s}$. The coefficients $c_{j}=W_{j} g_{j}, j=0, \ldots, M-1$ characterize the filter impulse response. Coefficients $W_{j}$ depend on the integration method; for the trapezoidal method they are given by: $W_{0}=W_{M}=\frac{1}{2 M}, W_{j}=\frac{1}{M}, j=1, \ldots, M-1$. When $\mu, \kappa$ are negative better results are obtained (noise effect and delay are reduced), but in that case one have to modify $W_{0}=W_{M}$ (see [3]). Coefficients $g_{j}$ are such as $g_{j}=g_{\kappa, \mu, N}\left(\frac{j}{M}\right), j=0, \ldots, M$.

\section{MAin Results}

\section{A. Localizability study in the geometric framework}

Using (2)-(3) and the kinematic model (1) we have $d \alpha_{i}=$ $\frac{y_{r, i} d x-x_{r, i} d x}{x_{r, i}^{2}+y_{r, i}^{2}}-d \theta, d \beta_{i}=\frac{z_{A_{i}}\left(x_{r, i} d x+y_{r, i} d y\right)}{\sqrt{x_{r, i}^{2}+y_{r, i}^{2}}\left(x_{r, i}^{2}+y_{r, i}^{2}+z_{A_{i}}^{2}\right)}$, leading to (see Theorem 1):

$$
d \mathcal{O}_{\mathbf{x}}(\mathbf{h})=\mathcal{O}(d x, d y, d \theta)^{T}
$$

with $\operatorname{det} \mathcal{O}=\frac{z_{A_{i}}}{d_{2 D}\left(R, A_{i}\right) d_{3 D}\left(R, A_{i}\right)^{2}}$. This determinant is non zero and is defined if and only if $z_{A_{i}} \neq 0$ and $d_{2 D}\left(R, A_{i}\right) \neq 0$. So the rank of the observability codistribution is 3 under the same conditions.

Theorem 5: A unicycle mobile robot with kinematic model (1) which measures its orientation and the two angles between itself and a landmark is localizable (i.e. it is possible to estimate its posture $\left.(x, y, \theta)^{T}\right)$, if and only if:

- $d_{2 D}\left(R, A_{i}\right) \neq 0$, i.e. the projection of the relative distance between the robot and the landmarks in the plan of motion is nonzero,

- $z_{A_{i}} \neq 0$, i.e. the height of the landmark is non-zero.

\section{B. Algebraic estimation of the robot's posture and velocities}

Theorem 6: [1] For a unicycle mobile robot with kinematic model (1) with measurement model (2)-(3), the velocities (linear $u$ and angular $\omega$ ) and the relative coordinates of the robot $\left(x_{r, i}, y_{r, i}\right)^{T}$ are are estimated by:

$$
\begin{aligned}
\hat{u} & =\frac{z_{A_{i}} \dot{\beta}_{f, i}}{\sin ^{2} \beta_{f, i} \cos \alpha_{f, i}}, \\
\hat{\omega} & =\frac{2 \tan \left(\alpha_{f, i}\right)}{\sin \left(2 \beta_{f, i}\right)} \dot{\beta}_{f, i}-\dot{\alpha}_{f, i}, \\
\left(\begin{array}{c}
\hat{x}_{r, i} \\
\hat{y}_{r, i}
\end{array}\right) & =\left(\begin{array}{c}
\frac{z_{A_{i}} \cos \left(\alpha_{f, i}+\theta_{f}\right)}{\tan \left(\beta_{f, i}\right)} \\
\frac{z_{A_{i}} \sin \left(\alpha_{f, i}+\theta_{f}\right)}{\tan \left(\beta_{f, i}\right)}
\end{array}\right) .
\end{aligned}
$$

Proof: The proof for the linear speed and angular speed estimation can be found in [1]. For the posture estimation, we have (Fig. 1) $d_{2 D}\left(R, A_{i}\right)=\frac{z_{A_{i}}}{\tan \left(\beta_{i}\right)}$. Since $x_{r, i}=$ $d_{2 D}\left(R, A_{i}\right) \times \cos \left(\alpha_{i}+\theta\right), y_{r, i}=d_{2 D}\left(R, A_{i}\right) \times \sin \left(\alpha_{i}+\theta\right)$ the result is proven.

\section{Sensibility study}

The sensibility of an estimated $\hat{x}$ (14)-(16) with respect to a measured quantity $z$ is denoted $S_{\hat{x} / z}$ and is defined as the partial derivative of the estimate with respect to each 


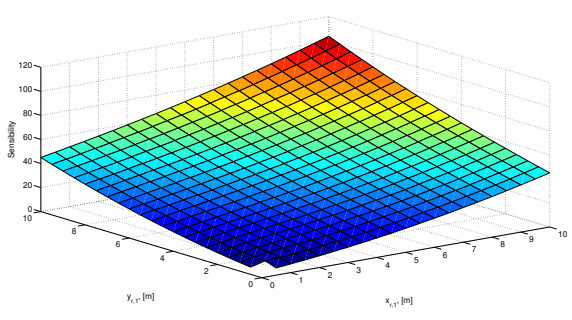

Fig. 2. CAS when $z_{A_{i}}=5 m$

measurement multiplied by the standard deviation of the corresponding measurement (denoted $\sigma_{z}$ ). This sensibility is clearly a function of the relative distance between the robot and the landmark:

$$
\begin{aligned}
S_{\hat{x}_{r, i} / \alpha_{f, i}} & =-y_{r, i} \sigma_{\alpha_{f, i}}, \\
S_{\hat{x}_{r, i} / \theta_{f}} & =-y_{r, i} \sigma_{\theta_{f}} \\
S_{\hat{x}_{r, i} / \beta_{f, i}} & =\frac{-d_{3 D}\left(R, A_{i}\right)^{2} x_{r, i}}{d_{2 D}\left(R, A_{i}\right) z_{A_{i}}} \sigma_{\beta_{f, i}} .
\end{aligned}
$$

Similarly, the sensibilities of the estimation of $y_{r, i}$ are:

$$
\begin{aligned}
S_{\hat{y}_{r, i} / \alpha_{f, i}} & =-x_{r, i} \sigma_{\alpha_{f, i}}, \\
S_{\hat{y}_{r, i} / \theta_{f}} & =-x_{r, i} \sigma_{\theta_{f}}, \\
S_{\hat{y}_{r, i} / \beta_{f, i}} & =\frac{-d_{3 D}\left(R, A_{i}\right)^{2} y_{r, i}}{d_{2 D}\left(R, A_{i}\right) z_{A_{i}}} \sigma_{\beta_{f, i}} .
\end{aligned}
$$

These relations show that the closer to the landmark the robot is, the better the solution is, except in the limit case when the robot is not localizable $\left(d_{2 D}\left(R, A_{i}\right)=0\right.$ or $\left.z_{A_{i}}=0\right)$. This could be shown by plotting the following equation which could be seen as the cumulative absolute sensibility (CAS):

$C A S=2\left|y_{r, i}\right|+2\left|x_{r, i}\right|+\left|\frac{d_{3 D}\left(R, A_{i}\right)^{2} y_{r, i}}{d_{2 D}\left(R, A_{i}\right) z_{A_{i}}}\right|+\left|\frac{d_{3 D}\left(R, A_{i}\right)^{2} x_{r, i}}{d_{2 D}\left(R, A_{i}\right) z_{A_{i}}}\right|$.

The evolution of CAS ( $z$-axis) with respect to $x_{r, i}=\left(x_{A_{i}}-\right.$ $x)$ (x-axis) and $y_{r, i}=\left(y_{A_{i}}-y\right)(y$-axis) is plotted on Fig. 2 for $z_{A_{i}}=5 \mathrm{~m}$.

\section{Fusion algorithm when there is more than one landmark}

When there is more than one landmark, each landmark can give an estimation of the posture by applying the result of Theorem 6. In this case, in order to give a final estimation of the posture, a fusion can be made. In [1], an average of the estimation given by each landmark is computed to get a global solution. Here, the sensibility is used to compute a weighted average of the solution given by each landmark: the proposed algorithm is summarized on Fig. 3. For each landmark $A_{i}$ at each time, an estimation of the relative position $\left(\hat{x}_{r, i}, \hat{y}_{r, i}\right)^{T}$ is computed and used to compute the sensibility of the current posture estimation with respect to the landmarks coordinates:

$$
\begin{aligned}
S_{\hat{x}_{r, i}} & =-\left(\hat{y}_{r, i} \sigma_{\theta}+\hat{y}_{r, i} \sigma_{\alpha_{i}}+\frac{\hat{d}_{3 D}\left(R, A_{i}\right)^{2} \hat{x}_{r, i}}{\hat{d}_{2 D}\left(R, A_{i}\right) z_{A_{i}}} \sigma_{\beta_{i}}\right),(19) \\
S_{\hat{y}_{r, i}} & =-\left(\hat{x}_{r, i} \sigma_{\theta}+\hat{x}_{r, i} \sigma_{\alpha_{i}}+\frac{-\hat{d}_{3 D}\left(R, A_{i}\right)^{2} \hat{y}_{r, i}}{\hat{d}_{2 D}\left(R, A_{i}\right) z_{A_{i}}} \sigma_{\beta_{i}}\right)(20)
\end{aligned}
$$

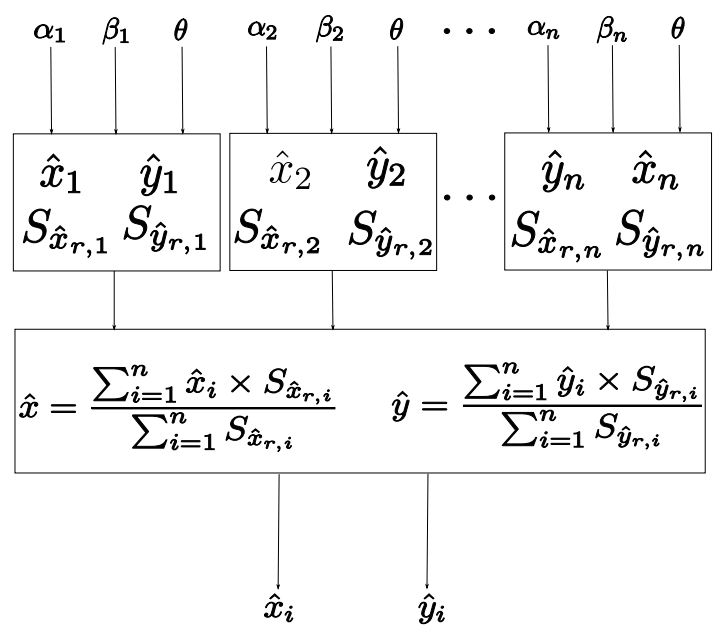

Fig. 3. Multi-landmarks localization using a mono-landmark approach

The relative position of robot with respect to each landmark $A_{i}$ denoted by $\left(\hat{x}_{r, i}, \hat{y}_{r, i}\right)^{T}$ can be used to compute the robot position denoted by $\left(\hat{x}_{i}, \hat{y}_{i}\right)^{T}$. Eventually by fusing all these estimates as follows we get the final pose estimation:

$$
\begin{aligned}
\hat{x} & =\frac{\sum_{i=i}^{n} \hat{x}_{i} \times S_{\hat{x}_{r, i}}}{\sum_{i=i}^{n} S_{\hat{x}_{r, i}}}, \\
\hat{y} & =\frac{\sum_{i=i}^{n} \hat{y}_{i} \times S_{\hat{y}_{r, i}}}{\sum_{i=i}^{n} S_{\hat{y}_{r, i}}} .
\end{aligned}
$$

\section{EXPERIMENTAL AND Simulation RESUlts}

\section{A. Simulation results}

In order to show the effectiveness of the proposed method, the obtained results have been implemented on Matlab. In order to give some comparison results in the multi-landmarks case, three algorithms have been implemented:

1) The original NON-A mean algorithm (mean of the solutions obtained for each landmark), see [1],

2) The proposed NON-A sensibility algorithm (weighted average of the solutions obtained for each landmark: the weight being the sensibility of the corresponding solution),

3) The EKF algorithm (as a reference in the robotics field, see [29] for example).

In [1] a theoretical comparison between the NON-A approach and the well known EKF approach has been given. Tab. I recalls the main points. The fundamental difference between NON-A mean algorithm and NON-A sensibility algorithm lies in the need for NON-A sensibility algorithm to know the statistical noise properties.

The algorithms are tested for 2, 6, 10, 50 and 100 landmarks. For each case, the simulation is repeated on 100 run and an uniformly distributed gaussian noise on the interval $\left[-0.5^{\circ} ; 0.5^{\circ}\right]$ is added to the angular measurements. The initial covariance matrix for the EKF is set at $0.1 \mathrm{~m}$ on the diagonal for the position and $1^{\circ}$ on the diagonal for the orientation and 0 elsewhere. The initial position is fixed at 


\begin{tabular}{|c|c|c|c|}
\hline The algorithm : & $\begin{array}{l}\text { NON-A } \\
\text { mean } \\
\text { algorithm }\end{array}$ & $\begin{array}{l}\text { NON-A } \\
\text { sensi- } \\
\text { bility } \\
\text { algorithm }\end{array}$ & EKF \\
\hline Needs to know the control input & NO & NO & YES \\
\hline Needs to know the noise characteristics & NO & YES & YES \\
\hline Needs to be initialized & NO & N0 & YES \\
\hline Gives an estimation of the control input & YES & YES & NO \\
\hline
\end{tabular}

TABLE I

COMPARISON OF THREE LOCALIZATION ALGORITHMS HYPOTHESIS, [1]

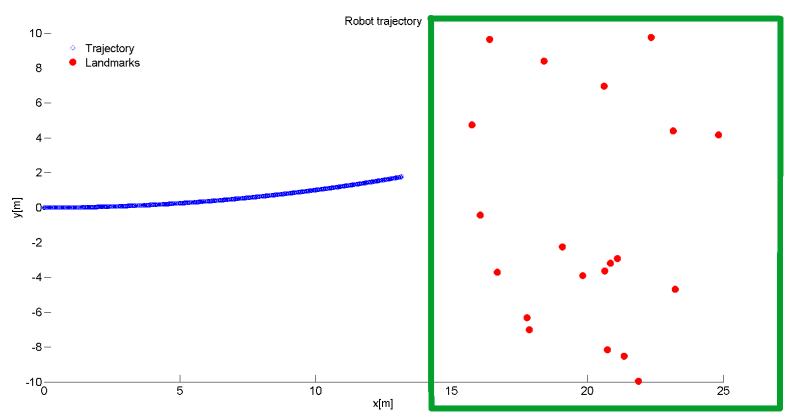

Fig. 4. Simulation with one block of landmarks

$10 \mathrm{~cm}$ from the true one for $x$ and $y$ and at $1^{\circ}$ for the initial orientation.

1) Simulation with one block of landmarks: Fig. 4 shows the trajectory used for the simulation. The position of the landmarks are chosen randomly at each run in a block (in green on the figure). In this first case, we use one block of landmarks. Tab. II gives the numerical results.

\begin{tabular}{|c|c|c|c|c|c|}
\hline number of landmarks & 1 & 5 & 10 & 50 & 100 \\
\hline$e_{N O N-A-M E A N}(\mathrm{~cm})$ & 1.6606 & 1.2131 & 1.0814 & 0.9422 & 0.9342 \\
\hline$e_{N O N-A-S E N S I B I L I T Y}(\mathrm{~cm})$ & 1.6032 & 1.1634 & 1.0446 & 0.9363 & 0.9332 \\
\hline$e_{E K F}(\mathrm{~cm})$ & 2.3552 & 1.8137 & 1.7197 & 1.3939 & 1.2210 \\
\hline$\sigma_{N O N-A-M E A N}\left(\mathrm{~cm}^{2}\right)$ & 0.1331 & 0.0360 & 0.0237 & 0.0203 & 0.0152 \\
\hline$\sigma_{N O N-A-S E N S I B I L I T Y}\left(\mathrm{~cm}^{2}\right)$ & 0.1215 & 0.0297 & 0.0204 & 0.0185 & 0.0140 \\
\hline$\sigma_{E K F}\left(\mathrm{~cm}^{2}\right)$ & 0.1510 & 0.0161 & 0.0121 & 0.0038 & 0.0018 \\
\hline$t_{N O N-A-M E A N}$ & 0.2706 & 0.4255 & 0.4721 & 0.4354 & 0.3863 \\
\hline$t_{N O N-A-S E N S I B I L I T Y}^{t_{E K F}}$ & (0) & 0 & 0 & 0432 & \\
\hline$t_{E K F}$ & & & & & \\
\hline
\end{tabular}

TABLE II

COMPARATIVE RESULTS BETWEEN THE THREE METHODS WITH ONE BLOCK OF LANDMARKS

We can notice that the two NON-A algorithms give equal or better results than the EKF algorithm. The main point of improvement lies in the computation time which is greatly reduced.

2) Simulation with two blocks of landmarks: Fig. 5 shows the trajectory used for the simulation. The position of the landmarks are chosen randomly at each run in a block (in green on the figure). In this second case, we use two blocks of landmarks. The second block (on the right of the figure) is far away from the robot and will give less accurate results than the first block (on the left of the figure). Tab. III gives the numerical results.

We can notice that NON-A sensibility algorithm is better

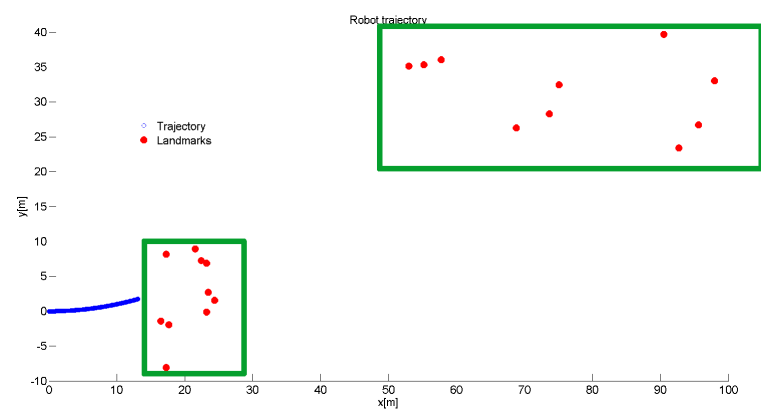

Fig. 5. Simulation with two blocks of landmarks

\begin{tabular}{|c|c|c|c|c|c|}
\hline number of landmarks & 2 & 6 & 10 & 50 & 100 \\
\hline \multirow{3}{*}{$\begin{array}{c}e_{N O N-A-M E A N}(\mathrm{~cm}) \\
e_{N O N-A-S E N S I B I L I T Y}(\mathrm{~cm}) \\
e_{E K F}(\mathrm{~cm}) \\
\end{array}$} & 15.2570 & 9.9732 & 7.6324 & 4.1055 & 3.3923 \\
\hline & 2.9550 & 1.8344 & 1.5591 & 1.1697 & 1.1406 \\
\hline & 3.2767 & 1.9699 & 1.7670 & 1.3647 & 1.2168 \\
\hline \multirow{3}{*}{$\begin{array}{c}\sigma_{N O N-A-M E A N\left(\mathrm{~cm}^{2}\right)} \\
\sigma_{N O N-A-S E N S I B I L I T Y}\left(\mathrm{~cm}^{2}\right) \\
\sigma_{E K F}\left(\mathrm{~cm}^{2}\right) \\
\end{array}$} & 60.2199 & 9.9514 & 3.4392 & 0.5371 & 0.2815 \\
\hline & 0.6881 & 0.1182 & 0.0832 & 0.0292 & 0.0285 \\
\hline & 1.1092 & 0.0428 & 0.0165 & 0.0026 & 0.0016 \\
\hline \multirow{3}{*}{$\begin{array}{l}\frac{t_{N O N-A-M E A N}}{t_{E K F}} \\
\frac{t_{N O N-A-S E N S I B I L I T Y}}{t_{N} K F}\end{array}$} & 0.3718 & 0.4081 & 0.4581 & 0.4286 & 0.3747 \\
\hline & 0.3104 & 0.3919 & 0.4231 & 0.4103 & 0.3621 \\
\hline & & & & & \\
\hline
\end{tabular}

TABLE III

COMPARATIVE RESULTS BETWEEN THE THREE METHODS WITH TWO BLOCKS OF LANDMARKS

than the NON-A mean algorithm in term of error mean and error variance. Furthermore, we can notice that NON-A sensibility algorithm gives better results than EKF algorithm.

\section{B. Experimental results}

We have implemented this algorithm on a real robot equipped with an Imaging Source camera and an Xsens IMU (see the left of Fig. 6). The IMU is only used to measure the orientation of the robot. The camera has been calibrated before the experiment in order to know its focal length. One image taken by the camera is shown on the right of Fig. 6 . The used landmark is a red point which is extracted from the video stream. The coordinate of the landmark is known. The goal of the experiment is to show the result given by NON-A algorithm with only one landmark.

The experiment has been made 10 times on the same trajectory (see Fig. 7). The results for NON-A algorithm are resumed in Tab. IV.

\begin{tabular}{|c|c|c|}
\hline & error mean $(\mathrm{cm})$ & error standard deviation $\left(\mathrm{cm}^{2}\right)$ \\
\hline NON-A & 9.38 & 3.4 \\
\hline
\end{tabular}

TABLE IV

EXPERIMENTAL RESULTS OF NON-A ALGORITHM

\section{CONCLUSION}

This paper first recalled the connection between localizability and observability notions in both frameworks (differential geometry and differential algebra). Then algebraic localizability criterium is given and used to provide a differential estimator as the one introduced in [1]. This paper gives 


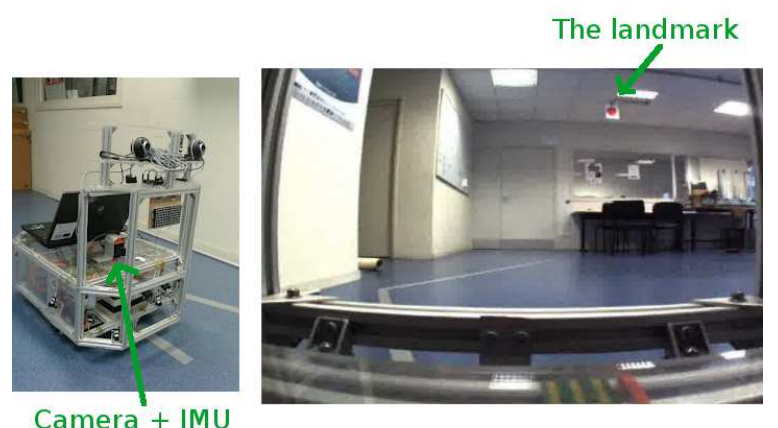

Fig. 6. The robot - An image of the camera

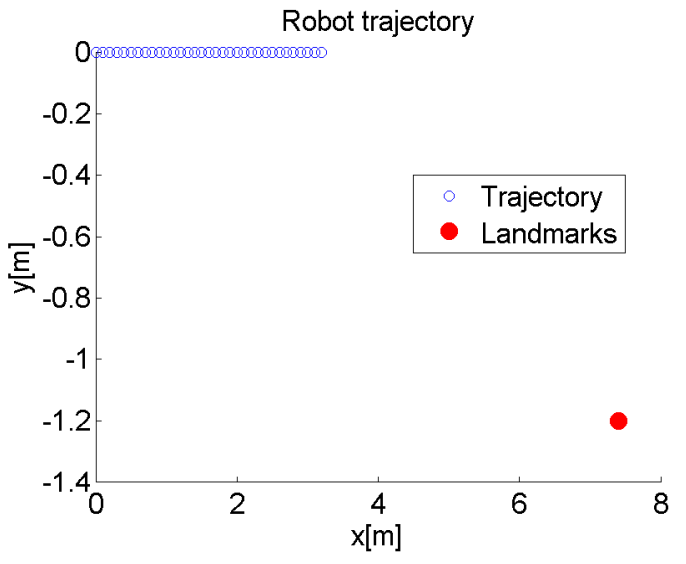

Fig. 7. Trajectory used for the experiment

a criterion to choose the landmarks position with respect to the robot trajectories. Lastly, in the multi-landmark case, we improve this algebraic localization algorithm by fusing the obtained estimates using a weighted mean: the weights are related to the sensibilities. This algorithm has been compared with the solution presented in [1], where a simple average between the estimations given by each landmark is used, as well as with the EKF. The simulation and experimental results show the effectiveness of the new proposed method with respect to both EKF and previous method, notably in term of computing time, mean and variance of error.

\section{REFERENCES}

[1] H. Sert, A. Kokosy, and W. Perruquetti, "A single landmark based localization algorithm for non-holonomic mobile robots," in IEEE International Conference on Robotics and Automation, 2011, pp. 293298.

[2] M. Mboup, C. Join, and M. Fliess, "Numerical differentiation with annihilators in noisy environment," Numerical Algorithms, vol. 50, pp. 439-467, 2009.

[3] D.-Y. Liu, O. Gibaru, and W. Perruquetti, "Error analysis of Jacobi derivative estimators for noisy signals," Numerical Algorithms, vol. 58, no. 1, pp. 53-83, Feb. 2011. [Online]. Available: http://hal.inria.fr/inria-00573270

[4] J. Leonard and H. Durrant-Whyte, "Mobile robot localization by tracking geometric beacons," Robotics and Automation, IEEE Transactions on, vol. 7, no. 3, pp. 376-382, 1991.

[5] J. Borenstein, H. Everett, and L. Feng, "Where am I? Sensors and methods for mobile robot positioning," University of Michigan, 1996.
[6] T. Lau, Y. Liu, and K. Lin, "A robust state estimation method against gnss outage for unmanned miniature helicopters," in IEEE International Conference onRobotics and Automation,, 2010, pp. 1116-1122.

[7] J. Levinson and S. Thrun, "Robust vehicle localization in urban environments using probabilistic maps," in IEEE International Conference on Robotics and Automation, 2010, pp. 4372-4378.

[8] J. Biswas and M. Veloso, "Wifi localization and navigation for autonomous indoor mobile robots," in IEEE International Conference on Robotics and Automation, 2010, pp. 4379-4384.

[9] A. Raghavan, H. Ananthapadmanaban, M. Sivamurugan, and B. Ravindran, "Accurate mobile robot localization in indoor environments using bluetooth," in IEEE International Conference on Robotics and Automation, 2010, pp. 4391-4396.

[10] W. You, B. Choi, B. Kim, H. Moon, J. Koo, W. Chung, and H. Choi, "Global localization for a small mobile robot using magnetic patterns," in IEEE International Conference on Robotics and Automation, , 2010, pp. 2618-2623.

[11] C. Harris and M. Stephens, "A combined corner and edge detector," in Alvey vision conference, vol. 15, 1988, p. 50.

[12] J. Shi and C. Tomasi, "Good features to track," in IEEE Computer Society Conference on Computer Vision and Pattern Recognition, 1994, pp. 593-600.

[13] D. Lowe, "Distinctive image features from scale-invariant keypoints," International journal of computer vision, vol. 60 , no. 2, pp. 91-110, 2004.

[14] H. Bay, T. Tuytelaars, and L. Van Gool, "Surf: Speeded up robust features," Computer Vision-ECCV, pp. 404-417, 2006.

[15] J. Font-Llagunes and J. Batlle, "Consistent triangulation for mobile robot localization using discontinuous angular measurements," Robotics and Autonomous Systems, vol. 57, no. 9, pp. 931-942, 2009.

[16] G. Jang, S. Kim, J. Kim, and I. Kweon, "Metric localization using a single artificial landmark for indoor mobile robots," in IEEE International Conference on Intelligent Robots and Systems, 2005, pp. 1992 $-1997$.

[17] T. Lemaire, S. Lacroix, and J. Sola, "A practical 3D bearing-only SLAM algorithm," in IEEE/RSJ International Conference on Intelligent Robots and Systems, 2005, pp. 2449-2454.

[18] P. Rudol, M. Wzorek, and P. Doherty, "Vision-based pose estimation for autonomous indoor navigation of micro-scale unmanned aircraft systems," in IEEE International Conference on Robotics and Automation, , 2010, pp. 1913-1920.

[19] A. Bais, R. Sablatnig, and J. Gu, "Single landmark based selflocalization of mobile robots," Computer and Robot Vision, Canadian Conference, p. 67, 2006.

[20] N. Dao, B. You, and S. Oh, "Visual navigation for indoor mobile robots using a single camera," in IEEE International Conference on Intelligent Robots and Systems, 2005.

[21] F. Conticelli, A. Bicchi, and A. Balestrino, "Observability and nonlinear observers for mobile robot localization," in IFAC International Symposium on Robot Control, 2000.

[22] A. Bicchi, D. Prattichizzo, A. Marigo, and A. Balestrino, "On the observability of mobile vehicles localization," in IEEE Mediterranean Conference on Control and Systems, 1998.

[23] A. Martinelli and R. Siegwart, "Observability analysis for mobile robot localization," in IEEE/RSJ International Conference on Intelligent Robots and Systems, 2005, pp. 1471-1476.

[24] G. Campion, G. Bastin, and B. Dandrea-Novel, "Structural properties and classification of kinematic and dynamic models of wheeled mobile robots," IEEE Transactions on Robotics and Automation, vol. 12, no. 1, pp. 47-62, 1996.

[25] R. Hermann and A. Krener, "Nonlinear controllability and observability," IEEE Transactions on Automatic Control,, vol. 22, no. 5, pp. 728-740, 1977.

[26] S. Diop and M. Fliess, "Nonlinear observability, identifiability, and persistent trajectories," in IEEE Conference on Decision and Control, Brighton, England, December 1991.

[27] _ , "On nonlinear observability," in Proceedings of European Control Conference, 1991, pp. 152-157.

[28] M. Fliess and H. Sira-Ramírez, "An algebraic framework for linear identification," ESAIM controle optimisation et calcul des variations, vol. 9, pp. 151-168, 2003.

[29] H. Sorenson, Kalman filtering: theory and application. IEEE Press, 1985. 